\title{
Exact solutions for transient mixing of two gases of different densities
}

Antonio L. Sánchez and Marcos Vera

Area de Mecánica de Fluidos, Universidad Carlos III de Madrid, 28911 Leganés, Spain

Amable Liñán

E. T. S. I. Aeronáuticos, Pl. Cardenal Cisneros 3, 28040 Madrid, Spain

(Received 10 February 2006; accepted 31 May 2006; published online 11 July 2006)

This Brief Communication presents a number of exact solutions describing the transient mixing of two gases of different molecular weights. Descriptions are given for both the concentration field and the associated induced motion in one-dimensional spherical, cylindrical, and planar configurations, including mixing layers, pockets, coflow jets, and concentrated mass sources. (C) 2006 American Institute of Physics. [DOI: 10.1063/1.2221349]

One-dimensional unsteady mixing of two different fluids is a fundamental problem that encounters many practical applications. ${ }^{1}$ When the density is constant, mixing by itself does not lead to fluid motion, so that the description of timedependent mixing in a quiescent fluid reduces to the integration of the transient heat equation, ${ }^{1}$ often leading to exact solutions in symmetric one-dimensional configurations (see, e.g., the extensive review given in Ref. 2). Variable density and its associated induced motion enter to complicate the description of mixing in gaseous mixtures. The changes in density may be associated with differences in temperature, as occurs for instance in the flow induced by heat release from localized energy sources, ${ }^{3}$ or may be associated with differences in molecular weight, the case considered herein.

We address in particular the transient one-dimensional evolution of an isothermal binary gas mixture. It is seen that the solution reduces to the integration of a linear parabolic equation for the density field. The equation further simplifies to the linear heat equation in a number of cases, including mixing of two semi-infinite gas spaces and mixing of a gas pocket with the ambient, for which exact analytical solutions are given. As explained in the text, these exact solutions apply also to the boundary-layer description of mixing in coflow jets when the velocity is uniform. The flow induced by localized mass sources of constant release rate is addressed next. We give in particular the self-similar exact solution for the line source in terms of the relevant Peclet number of the flow.

The one-dimensional isothermal mixing of two different gases of densities $\rho_{a}$ and $\rho_{b}$ is determined by integration of the continuity equation

$$
\frac{\partial \rho}{\partial t}+\frac{1}{r^{i}} \frac{\partial}{\partial r}\left(r^{i} \rho v\right)=0
$$

and the species transport equation

$$
\rho \frac{\partial Y}{\partial t}+\rho v \frac{\partial Y}{\partial r}=\frac{1}{r^{i}} \frac{\partial}{\partial r}\left(r^{i} \rho D \frac{\partial Y}{\partial r}\right)
$$

supplemented with the equation of state of an ideal gas written for a constant-temperature isobaric binary mixture

$$
Y=\frac{\rho_{a}\left(\rho_{b}-\rho\right)}{\rho\left(\rho_{b}-\rho_{a}\right)} .
$$

Here, $\rho$ and $v$ represent the density and velocity of the gas mixture, $Y$ is the mass fraction of the gas of density $\rho_{a}$, and $t$ and $r$ denote the time and the spatial coordinate, respectively. The index $i$ has been used for the plane, cylindrical, and spherical geometries. Note that relative pressure differences, which can be anticipated to be of the order of the square of the Knudsen number of the flow, have been neglected when writing (3). Correspondingly, baroclinic diffusion has been neglected in the multicomponent diffusion equation, ${ }^{4}$ which reduces exactly ${ }^{5}$ to the familiar Fick's law $Y V=-D(\partial Y / \partial r)$ used in (2), where $V$ and $D$ represent, respectively, the diffusion velocity of the gas of density $\rho_{a}$ and the binary diffusion coefficient of the two chemical species.

Since the temperature is uniform, changes in density and changes in composition are related by $\rho_{a} \rho_{b} \mathrm{~d} \rho=\left(\rho_{a}\right.$ $\left.-\rho_{b}\right) \rho^{2} d Y$, obtained by differentiating (3). Substitution of this equation into (2) yields

$$
\frac{\partial \rho}{\partial t}+v \frac{\partial \rho}{\partial r}=\frac{\rho}{r^{i}} \frac{\partial}{\partial r}\left(r^{i} \frac{D}{\rho} \frac{\partial \rho}{\partial r}\right),
$$

which, after subtraction of (1), reduces to

$$
\frac{\partial}{\partial r}\left[r^{i}\left(v+\frac{D}{\rho} \frac{\partial \rho}{\partial r}\right)\right]=0,
$$

finally giving

$$
r^{i}\left(v+\frac{D}{\rho} \frac{\partial \rho}{\partial r}\right)=q(t),
$$

where $q(t)$ is a function, with dimensions of a volumetric flux, to be determined from the boundary conditions. Substitution of this equation into (1) reduces the problem to that of integrating

$$
\frac{\partial \rho}{\partial t}-\frac{1}{r^{i}} \frac{\partial}{\partial r}\left(r^{i} D \frac{\partial \rho}{\partial r}-q \rho\right)=0
$$

with appropriate initial and boundary conditions. Once the above linear problem is solved for the density, one can use 
(3) and (6) to determine, respectively, the distributions of mass fraction and velocity.

The volumetric flux $q(t)$ appearing in (6) is identically zero in a number of cases of interest, for which the equation for the density (7) reduces to the well-known linear heat equation

$$
\frac{\partial \rho}{\partial t}=\frac{1}{r^{i}} \frac{\partial}{\partial r}\left(r^{i} D \frac{\partial \rho}{\partial r}\right) .
$$

One example of such solutions is the interdiffusion of two semi-infinite gas spaces of densities $\rho_{a}$ and $\rho_{b}$, which is described by integrating (8) for $i=0$ with initial condition $\rho$ $=\rho_{a}$ for $r<0$ and $\rho=\rho_{b}$ for $r>0$, and with boundary conditions $\rho=\rho_{a}$ as $r \rightarrow-\infty$ and $\rho=\rho_{b}$ as $r \rightarrow \infty$. The problem admits the self-similar solution

$$
\frac{\rho-\rho_{b}}{\rho_{a}-\rho_{b}}=\frac{1}{2}\left[1-\operatorname{erf}\left(\frac{r}{2(D t)^{1 / 2}}\right)\right],
$$

where erf is the error function. Note that this solution gives also the boundary-layer description for the density distribution across a mixing layer with uniform streamwise velocity, with the time $t$ representing in this case the distance to the origin of the mixing layer divided by the flow velocity.

The volumetric flux also vanishes when considering the diffusion of a gas pocket of radius $a$ into an infinite atmosphere of a different gas. The initial conditions for (8) become $\rho=\rho_{a}$ for $r<a$ and $\rho=\rho_{b}$ for $r>a$, whereas the boundary conditions for $t>0$ are given by $(\partial \rho) /(\partial r)=0$ at $r=0$ and $\rho=\rho_{b}$ as $r \rightarrow \infty$. Integration of (8) yields in this case ${ }^{2}$

$$
\frac{\rho-\rho_{b}}{\rho_{a}-\rho_{b}}=\frac{1}{2}\left[\operatorname{erf}\left(\frac{r+a}{2(D t)^{1 / 2}}\right)-\operatorname{erf}\left(\frac{r-a}{2(D t)^{1 / 2}}\right)\right]
$$

for the planar pocket $i=0$,

$$
\frac{\rho-\rho_{b}}{\rho_{a}-\rho_{b}}=\frac{1}{2 D t} e^{-r^{2} /(4 D t)} \int_{0}^{a} e^{-s^{2} /(4 D t)} I_{0}\left(\frac{r s}{2 D t}\right) s d s
$$

for the cylindrical pocket $i=1$, with $I_{0}$ representing the modified Bessel function of order zero, ${ }^{6}$ and

$$
\begin{aligned}
\frac{\rho-\rho_{b}}{\rho_{a}-\rho_{b}}= & \frac{1}{2}\left[\operatorname{erf}\left(\frac{r+a}{2(D t)^{1 / 2}}\right)-\operatorname{erf}\left(\frac{r-a}{2(D t)^{1 / 2}}\right)\right] \\
& -\frac{(D t)^{1 / 2}}{\sqrt{\pi} r}\left(e^{-(r-a)^{2} /(4 D t)}-e^{-(r+a)^{2} /(4 D t)}\right)
\end{aligned}
$$

for the spherical pocket $i=2$. It should be noted that the above results are also applicable to the description of the mixing of a gas jet of radius $a$ discharging into a coflow stream of a different gas, when the velocity of the jet $U$ is equal to that of the coflow stream and the relevant Peclet number $U a / D$ is sufficiently large for the boundary-layer approximation to apply. In that case, the problem reduces to that of gas-pocket diffusion, so that (10) and (11) give the density distributions across the plane and round jet, with $t$ representing in this case the distance to the jet exit divided by the uniform flow velocity.

As previously mentioned, straightforward substitution of (9)-(12) into (3) provides the mass fraction field, while the velocity field is given in this case by $v=-(D / \rho)(\partial \rho / \partial r)$. This last expression reveals that in isothermal jets with uniform axial velocity the transverse velocity vanishes outside the mixed region, implying that jet discharge produces no displacement of the ambient gas.

Nonzero values of $q$ appear for instance when analyzing the flow induced by the sudden injection of a gas of density $\rho_{a}$ from a concentrated source embedded in an initially quiescent infinite atmosphere of a different gas of density $\rho_{b}$. The corresponding boundary conditions at the source are

$$
\dot{m}_{i}=2^{i} \pi^{\delta_{i}}{ }^{i} \rho v=2^{i} \pi^{\delta_{i}} r^{i}\left(\rho v Y-\rho D \frac{\partial Y}{\partial r}\right),
$$

as $r \rightarrow 0$, where $\delta_{i}=0$ for $i=0$ and $\delta_{i}=1$ for $i \neq 0$. Here, $\dot{m}_{i}$ represents the mass release rate per unit surface for a planar source $(i=0)$, the mass release rate per unit length for a line source $(i=1)$, and the mass release rate for a point source $(i=2)$. Equations (13) state that, at the source, both the mass flow rate and the mass rate of the gas of density $\rho_{a}$ equal the known mass release rate $\dot{m}_{i}$, which in principle can be a function of time. Combining (13) with (3) yields the boundary condition

$$
r^{i} D \frac{\partial \rho}{\partial r}=\frac{\dot{m}_{i}}{2^{i} \pi^{\delta_{i}} \rho_{a}}\left(\rho-\rho_{a}\right)
$$

for the density as $r \rightarrow 0$ and the value of the function

$$
q=\frac{\dot{m}_{i}}{2^{i} \pi^{\delta_{i}} \rho_{a}}
$$

The problem reduces to that of integrating (7) with initial condition $\rho=\rho_{b}$ at $t=0$ and with boundary conditions for $t$ $>0$ given by (14) as $r \rightarrow 0$ and by $\rho \rightarrow \rho_{b}$ as $r \rightarrow \infty$.

It can be seen that the above problem admits a selfsimilar solution when $\dot{m}_{i}$ is of the form $\dot{m}_{i} \propto t^{(i-1) / 2}$. We consider below only the solution for constant release rate, which is self-similar for the line source $i=1$. Introducing the coordinate $\eta=r /(D t)^{1 / 2}$ and the dimensionless density difference $\phi=\left(\rho-\rho_{b}\right) /\left(\rho_{a}-\rho_{b}\right)$ produces the similarity problem

$$
\frac{\mathrm{d}^{2} \phi}{\mathrm{d} \eta^{2}}+\left(\frac{\eta}{2}-\frac{\mathrm{Pe}-1}{\eta}\right) \frac{d \phi}{d \eta}=0
$$

with boundary conditions

$$
\begin{aligned}
& \eta \rightarrow 0: \quad \eta(d \phi / d \eta)=\operatorname{Pe}(\phi-1), \\
& \eta \rightarrow \infty: \quad \phi \rightarrow 0,
\end{aligned}
$$

where $\mathrm{Pe}=\dot{m}_{1} /\left(2 \pi \rho_{a} D\right)$ is the relevant Peclet number of the flow. Integration yields

$$
\begin{aligned}
\frac{\rho-\rho_{b}}{\rho_{a}-\rho_{b}} & =1-\frac{1}{\Gamma(\mathrm{Pe} / 2)} \int_{0}^{r^{2} /(4 D t)} x^{\mathrm{Pe} / 2-1} e^{-x} d x \\
& =\Gamma\left(\frac{\mathrm{Pe}}{2}, \frac{r^{2}}{4 D t}\right) / \Gamma(\mathrm{Pe} / 2),
\end{aligned}
$$


where $\Gamma(\cdot)$ is the incomplete gamma function. ${ }^{6}$ In this case, and also in the spherico-symmetrical case $i=2$, diffusion becomes negligible as the source is approached, so that the density at the source is equal to $\rho_{a}$ from the initial instant. No exact solutions exist for the point and planar sources with constant mass release rate. For the planar source, a straightforward order-of-magnitude analysis of (7) indicates that $\rho_{a} D / \dot{m}_{0}$ and $\rho_{a}^{2} D / \dot{m}_{0}^{2}$ are the appropriate scales for length and time, while for the point source one obtains $\dot{m}_{2} /\left(4 \pi \rho_{a} D\right)$ and $\left[\dot{m}_{2} /\left(4 \pi \rho_{a}\right)\right]^{2} / D^{3}$. When these scales are used, the problem reduces to that of integrating a parameter-free parabolic equation that describes the evolution of the density around the source.

We have presented some exact solutions that describe the isothermal mixing of two different gases in onedimensional symmetrical configurations, including the interdiffusion of two semi-infinite spaces and the isovelocity mixing layer, given in (9), the diffusion of a gas pocket and the isovelocity jet, given in (10)-(12), and the line mass source of constant rate, given in (17). Besides their value as solu- tions to fundamental problems, the above exact expressions may find application in benchmarking exercises of numerical codes.

This collaborative research was supported by the Spanish MEC under Project Nos. ENE2005-08580-C02-01 and ENE2005-09190-C04-01 and by the Comunidad de Madrid under Project No. OS-505/ENE/0229.

${ }^{1}$ J. Crank, The Mathematics of Diffusion, 2nd ed. (Oxford University Press, Oxford, 1993).

${ }^{2}$ H. S. Carslaw and J. C. Jaeger, Conduction of Heat in Solids, 2nd ed. (Oxford University Press, Oxford, 1959).

${ }^{3}$ A. L. Sánchez, J. L. Jiménez-Alvarez, and A. Liñán, "The coupling of motion and conductive heating of a gas by localized energy sources," SIAM J. Appl. Math. 63, 937 (2003).

${ }^{4}$ J. O. Hirschfelder, C. F. Curtiss, and R. B. Bird, Molecular Theory of Gases and Liquids (Wiley, New York, 1954).

${ }^{5}$ F. A. Williams, Combustion Theory, 2nd ed. (Benjamin Cummins, Menlo Park, CA, 1985)

${ }^{6}$ M. Abramowitz and I. A. Stegun, Handbook of Mathematical Functions (Dover, New York, 1965). 
Physics of Fluids is copyrighted by the American Institute of Physics (AIP). Redistribution of journal material is subject to the AIP online journal license and/or AIP copyright. For more information, see http://ojps.aip.org/phf/phfor.jsp 